\title{
Integrated analysis of whole genome and transcriptome sequencing in a young patient with gastric cancer provides insights for precision therapy
}

\author{
KONGWANG HU ${ }^{1}$, WEIQIANG YU ${ }^{2}$, OLUGBENGA EMMANUEL AJAYI ${ }^{2}$, \\ LONGLONG LI $^{1}$, ZHIGUO HUANG ${ }^{1}$, QIQI RONG ${ }^{2}$, SHUAILI WANG ${ }^{2}$ and QING-FA WU ${ }^{2}$ \\ ${ }^{1}$ Division of Gastrointestinal Surgery, Department of General Surgery, The First Affiliated Hospital of \\ Anhui Medical University, Hefei, Anhui 230022; ${ }^{2}$ Hefei National Laboratory for Physical Sciences at Microscale, \\ CAS Key Laboratory of Innate Immunity and Chronic Disease, School of Basic Medical Sciences, Division of Life Sciences and \\ Medicine, University of Science and Technology of China, Hefei, Anhui 230026, P.R. China
}

Received June 4, 2019; Accepted July 17, 2020

DOI: 10.3892/ol.2020.11976

\begin{abstract}
Gastric cancer is a leading cause of cancerassociated deaths worldwide and is considered to be an age-related disease. In younger patients, gastric cancer is biologically more aggressive, and prognosis is worse compared with that in elderly patients. In the present case report, the whole genome and transcriptome was sequenced in a 26 -year-old patient with gastric cancer who presented with gastric cancer-related symptoms and was admitted to the First Affiliated Anhui Medical Hospital (Hefei, China) in December 2016. In total, 9 germline and 4 somatic mutations were identified in the patient, and there were more deleterious sites in the germline mutated genes. Genes with somatic mutations, such as $M U C 2, M U C 4, S L C 8 A 2$, and with structural variations, including $C C N D 3, F G F R 2$ and $F G F R 3$, were found to be differentially expressed. Cancer-associated pathways, such as the 'calcium signaling pathway', 'cGMP-PKG signaling pathway' and 'transcriptional mis-regulation' were also enriched at both the genomic and transcriptomic levels. The genes found to have germline (SFRP4), somatic (MUC2,
\end{abstract}

Correspondence to: Professor Qing-Fa Wu, Hefei National Laboratory for Physical Sciences at Microscale, CAS Key Laboratory of Innate Immunity and Chronic Disease, School of Basic Medical Sciences, Division of Life Sciences and Medicine, University of Science and Technology of China, 443 HuangShan Road, Hefei, Anhui 230026, P.R. China

E-mail:wuqf@ustc.edu.cn

Abbreviations: GC, gastric cancer; CGC, Cancer Gene Census; CPG, Cancer Predisposition Genes; Indels, insertions and deletions; SNP, single nucleotide polymorphism; SNV, single nucleotide variation; GO, Gene Ontology; KEGG, Kyoto Encyclopedia of Genes and Genomes; TCGA STAD, The Cancer Genome Atlas stomach adenocarcinoma

Key words: gastric cancer, young age, germline mutations, somatic mutations, TCGA STAD
MUC4, SLC8A2) mutations, or structural variations (CCND3, $F G F R 2$ and $F G F R 3$ ) were differentially expressed in the patient and could be promising precision therapy targets.

\section{Introduction}

Gastric cancer (GC) is a leading cause of cancer-related deaths worldwide and is known to be an age-related disease (1). The mean age of patients with GC at diagnosis is $\sim 60$ years and $<3 \%$ of GC cases are reported in patients $<30$ years of age $(2,3)$. Several reports have found that younger patients ( $\sim 30$ years) are often diagnosed with advanced stages of GC and have worse prognosis compared with that in elderly patients ( 60 years) $(4,5)$. In younger patients, the cancer was found to spread rapidly and was biologically more aggressive (6). Integrative genomic approaches, which associate genomic and transcriptomic analysis have been found to increase the identification of numerous events and processes associated with tumor aggressiveness and may assist in selecting candidate genes from normal and pathological samples for targeted therapy (7). A previous study aimed to investigate the biological and genomic mechanisms in the progression of GC has led to the development of target-oriented therapy for advanced GC to be used in a clinical setting, with base substitution mutations in genes such as tumor protein p53 (TP53), AT-rich interaction domain 1A (ARIDIA), KRAS proto-oncogene, GTPase $(K R A S)$, phosphatidylinositol-4,5-bisphosphate 3-kinase catalytic subunit alpha (PIK3CA), ring finger protein 43 (RNF43), APC regulator of WNT signaling pathway $(A P C)$, RAS p21 protein activator 1 (RASAl) and erb-b2 receptor tyrosine kinase 2 (ERBB2) identified as important therapeutic targets in anti-cancer treatments (8). Developing personalized therapy for optimal individual cancer patient outcome has become more feasible due to the rapid advancement in next-generation sequencing techniques and technologies that enable fast and comprehensive characterizations of tumors at the molecular level (9-12). As every patient harbors a unique combination of variants that influence the risk, onset, and progression of the disease, an effective personalized therapy is dependent on a 
well-profiled genome from the individual patient with cancer and understanding the oncogenic mechanisms that regulate the progression of the tumor (13). With a view to identifying potential clinically actionable therapeutic targets that may inform individualized treatment strategies, the whole genome was sequenced in a 26-year-old patient with advanced stage $\mathrm{GC}$, by integrating the whole genome and whole transcriptome sequencing data.

\section{Materials and methods}

Sample collection and preservation. A 26-year-old female patient was admitted to the First Affiliated Anhui Medical Hospital in December 2016 having presented with GC-related symptoms. The patient felt uncomfortable in the upper left quadrant of her abdomen and had repeated black melena for one month. This symptom recurred and became more serious for 2 weeks. The patient felt full and uncomfortable in the upper left quadrant of her abdomen repeatedly following a meal, one month prior to the development of black melena. She also had nausea, although there was no hematemesis or hematochezia. The abdominal discomfort worsened and the patient received treatment in Luan Jinkai Hospital. Thereafter, the patient visited the First Affiliated Hospital of Anhui Medical University where the present study was conducted. The gastroscopy of the patient indicated GC with obstruction, while pathology indicated antrum considered as carcinoma mucocellulare. Pathological examination of the samples was performed using hematoxylin and eosin staining. Briefly, tissues were sliced with a dimension of $1.5 \times 1.5 \times 0.2-0.3 \mathrm{~cm}$ and soaked in $40^{\circ} \mathrm{C}$ warm water. The sections were deparaffinized in xylene and were gradually hydrated through graded ethanol $(100,95,80$ and $70 \%)$ each for $3 \mathrm{~min}$ at $25^{\circ} \mathrm{C}$. The sections were stained in hematoxylin solution for $10 \mathrm{~min}$ at $25^{\circ} \mathrm{C}$ and differentiated in $1 \%$ hydrochloric alcohol. Then the slides were rinsed with tap water and distilled water until the nuclei became blue, and dehydrated in $95 \%$ ethanol. The sections were counterstained in $1 \%$ eosin solution for $5 \mathrm{~min}$ at $25^{\circ} \mathrm{C}$, washed with $70 \%$ ethanol twice and absolute ethanol, and were cleared in two washes of xylene. The sections were mounted with neutral balsam and observed under a light microscope magnification, x400 (x40 objective and x10 ocular magnification; BX-42; Olympus). An abdominal computer tomography scan + enhancement showed that both armpits had multiple small lymph nodes and the gastric antrum had thickened walls along with multiple swollen lymph nodes in the surrounding area. The patient was diagnosed with a T3N3bM0 (advanced stage IIIC) GC using AJCC TNM staging system 7th edition (14).

The patient underwent a gastrostomy for tumor removal. Tumor tissue and adjacent normal tissue $\sim 5-\mathrm{cm}$ away from the tumor were resected and preserved in liquid nitrogen until use.

DNA and RNA extraction and library preparation. Genomic DNA was isolated from the resected tissues using a genomic DNA isolation kit (Bioo Scientific; http://www.biooscientific. com) according to the manufacturer's recommendations. Extracted DNA was quantified using a NanoDrop ND-1000 spectrophotometer (NanoDrop Technologies; Thermo Fisher Scientific, Inc.) and the integrity was assessed using
$1 \%$ agarose gel electrophoresis and visualized in gel imager (Tanon; http://www.biotanon.com). RNA was extracted from the tumor and adjacent normal tissue using TRIzol ${ }^{\circledR}$ (Invitrogen; Thermo Fisher Scientific, Inc.), and eluted in RNAse-free water. RNA quantity and quality were assessed using an Agilent 2100 Bioanalyzer (Agilent Technologies Inc.). DNA and RNA libraries were prepared according to standard protocols according to Bioo Scientific.

Whole transcriptome sequencing and pre-analysis. For whole transcriptome sequencing, raw sequencing reads were produced using an Illumina X10 sequencer (Illumina Inc.) with 150-bp paired-end reads according to the manufacturer's instructions. Raw RNA-Sequencing (RNA-Seq) data was trimmed by removing the adaptors and low quality reads using trim_galore v0.4.4 software (15). The data was subsequently aligned to the human reference genome GRCh37.p13 using STAR v2.6.1a software (16) in a 2-pass mapping. Read count in the tumor and adjacent normal tissues was calculated using htseq-count v0.10.0 software (17), and differentially expressed genes were detected using edgeR v3.24.3 software (18). Genes with $\mathrm{P} \leq 0.001$ and $\log _{2}$ fold change $\geq 1$ were considered as differentially expressed.

Whole genome sequencing and pre-analysis. Whole genome sequencing of the prepared libraries was performed using the Illumina X10 sequencer (Illumina Inc.) with 150-bp paired-end reads according to the manufacturer's instructions. The raw fastq data was mapped to the human reference genome (b37) provided by the Broad Institute with the Burrows-Wheeler Aligner v.7.0.12 (19). Read duplications were marked using the Picard tool v.2.10.10; local realignment and base quality score recalibration was performed using Genome Analysis ToolKit (GATK) v. 3.8-0 (20) and the final bam files were used for variant calling.

Somatic and germline variants detection. Germline variants were called using the GATK HaplotyperCaller joint v3.8 software (21) and then filtered using GATK variant quality score recalibration (VQSR). Variants that passed using the VQSR module with coverage $\geq 6 \mathrm{X}$ and supporting reads $\geq 0$ were retained, while the variants showing genotype ' $0 / 0$ ' or '.l' in the tumor sample were filtered out. For somatic variant calling, three different types of software were used: GATK HaplotypeCaller joint v3.8, Mutect v1.1.4 and MuTect2 v3.8 (22). In Mutect and MuTect2, all variants that were flagged as 'PASS' were kept. Other variants were retained with a coverage $\geq 5 \mathrm{X}$ and supporting $\geq 3$ reads, if they were flagged among a subset of 'alternative (23) allele in normal', 'triallelic site', 'possible contamination', 'clustered read position' in Mutect, or 'germline risk', 'alt allele in normal', 't lod fstar' (tumor does not meet likelihood threshold), 'str contraction' (site filtered due to contraction of short tandem repeat region), 'triallelic site' (site filtered because > two alternate alleles pass tumor likelihood threshold) in MuTect2. Germline variants were removed from both the Mutect and MuTect2 output data. In all three softwares, variant allele frequency in the tumor sample had to be 3 times higher compared with that in the normal sample, and $>5 \%$ of the variant reads in the tumor sample was required. Functional annotations for both germline and somatic variants were added to each mutation using the 
ANNOVAR software v.2018Apr16 (17) and several publicly available databases, such as 1,000 Genome Project (24), the Exome Aggregation Consortium (25), the Genome Aggregation Database (26) and the compiled scores prediction system dbnsfp33a (27), dbSNP (28) and COSMIC (29).

Transition to transversion ratio calculation. The transition to transversion ratio for germline variants was calculated by dividing total transitions by total transversions. Transitions refer to variations that involve a change from purine to purine, while transversions refer to variations that involve a change from purine to pyrimidine or vice versa (30). The transition to transversion ratio between homologous strands of DNA is generally $\sim 2$, and for human, the ratio is $\sim 2.1$ (31).

Copy number alternation and structural variation analysis. Copy number variants (CNV), tumor purity and ploidy was analyzed using cnv_facets software (https://github.com/dariober/cnv_facets) based on FACETS v0.5.14 (32), and run with the '-cval 25 400' command. Duplicated and deleted segments were retained and annotated using an ENCODE gene symbol in R v3.5.1 (33). iCallSV (https://github.com/rhshah/iCallSV), which applied Delly v0.7.5 prediction method (34) was used to detect structural variations and fusions using default parameters. Inter- and intra-chromosomal fusions $>50 \mathrm{~kb}$ were retained and only in-frame deletions, duplications and fusions were selected for subsequent analysis.

Mutation significance analysis. The 1,000 Genome Project (24), the Exome Aggregation Consortium (25) and the Genome Aggregation Database (26) provided alternative allele frequency data in different populations that ANNOVAR software applied to the present variations. Annotations using a set of algorithm scoring system from dbNSFP v.33a, including FATHMM, LRT, Mutation Assessor, Mutation Taster, M-CAP, Polyphen2, PROVEAN and SIFT (35), were also applied to the variations. Then the common variants were filtered out, which are sites with a minor allele frequency $\geq 1 \%$, to get the most informative ones. Mutations predicted to be deleterious by at least two algorithms of dbNSFP were considered to be potential pathogenic sites of these informative variants.

Identification of mutations in cancer associated genes. To identify genes with a high possibility of involvement in cancer development, the Cancer Gene Census (CGC) (https:// cancer.sanger.ac.uk/census) (36) and Cancer Predisposition Genes (CPG) (37) databases were used. In total, 850 candidate genes from cancer-associated databases ( 723 genes from CGC and 114 genes from CPG with 87 overlapping genes), were investigated. The gene lists were downloaded directly from the two databases with no specific selection criteria.

Pathway analysis. Gene enrichment was performed using the R package clusterProfiler v3.10.1 software (38), which implements a hypergeometric model to test for gene set overrepresentation relative to a background gene set. Enrichment was analyzed using the functions: 'Enriched Kyoto Encyclopedia of Genes and Genomes (KEGG)', 'gseKEGG' (Gene Set Enrichment Analysis (GSEA) (39) of KEGG, and a function of clusterProfiler), $\mathrm{P}<0.05, \mathrm{Org} \mathrm{Db}=$ org.Hs.eg.db (a database for Genome Wide Annotation for Human, referenced by clusterProfiler).

Integrated analysis of whole genome and transcriptome sequencing data. The differentially expressed genes were compared with mutated genes identified by whole genome sequencing. To achieve this, genes that were mutated and with differentially expressed transcripts were merged in R v3.5.1 (33).

Analysis of public GC RNA-Seq data. The RNA-Seq data from The Cancer Genome Atlas stomach adenocarcinoma (TCGA STAD) was downloaded from TCGAbiolinks online tool (https://github.com/BioinformaticsFMRP/TCGAbiolinks). To identify the most significantly differentially expressed genes in GC, the results from three RNA-Seq differential expression analysis tools, including limma v3.42.2 (40), DEseq2 v1.26.0 (41) and edgeR v3.28.1 (18), were combined and the overlapping genes were separated for further analysis. For all three tools, $\mathrm{P}<0.05$ and the average $\log _{2}$ fold change \pm standard deviation were used as the cut-off values. Gene Ontology (42) enrichment analysis was performed using the Database for Annotation, Visualization and Integrated Discovery (DAVID) v6.7 functional annotation clustering tool to determine the potential functions of the differentially expressed genes. The KEGG pathway analysis was performed to determine the involvement of differentially expressed genes in different biological pathways. The- $\log 10(\mathrm{P})$ denotes the enrichment score, indicating the significance of the pathway associations.

Kaplan-Meier plotter (KM-Plotter) analysis. KM-plotter (https://kmplot.com/analysis/) is an online database and tool which can be used for the discovery and validation of survival biomarkers (43). It contains cancer gene expression data and survival information from Gene Expression Omnibus (44), European Genome-phenome Archive (EGA) (https://www. ebi.ac.uk/ega/home), and TCGA (https://www.cancer.gov/ tcga), and uses each percentile of mRNA expression between the lower $(25 \%)$ and upper quartiles (75\%) of expression as a cut-off point to divide patients into high and low expression groups. The KM-plotter software, which included available transcriptome and survival data (both overall and disease-free survival times), was used to analyze a total of 1,440 patients with GC, based on default parameters.

\section{Results}

Tumor purity and coverage statistics. The proportion and the ploidy of tumor cells in the sample were $\sim 22 \%$ and 1.74 , respectively. Using whole genome sequencing, the total number of paired reads were 8.13 and 8.34 billion, the mean read coverage was 37.16 and 35.48 , while the mean library insert size was 319 and 323 bp for normal and tumor tissue samples, respectively. For the consensus coding sequence region, $90 \%$ of the bases had over $30 \mathrm{X}$ coverage. Using whole transcriptome sequencing, 1.04 and 1.11 billion clean reads were obtained with an average input read length of 253 and $261 \mathrm{bp}$ for normal and tumor tissue, respectively.

Gene expression analysis. The RNA-Seq pipeline was used (Fig. S1A) for the whole transcriptome sequencing. Genes 
Table I. Functional assessment of the germline mutations and prediction of their effect.

\begin{tabular}{|c|c|c|c|c|c|c|c|c|c|}
\hline Gene & Databse & Chromosome & Position & $\begin{array}{l}\text { Reference } \\
\text { allele }\end{array}$ & Alteration & Type & $\begin{array}{c}\text { AA. } \\
\text { Change }\end{array}$ & Cosmic82 & $\begin{array}{c}\text { Final } \\
\text { prediction }\end{array}$ \\
\hline AKAP9 & CGC & 7 & 91739445 & $\mathrm{C}$ & $\mathrm{T}$ & Non-SNV & p.T3899I & $\mathrm{N}$ & $\mathrm{U}$ \\
\hline DOCK8 & CPG & 9 & 286491 & G & A & Non-SNV & p.D63N & $\mathrm{Y}$ & $\mathrm{D}$ \\
\hline HIF1A & CGC & 14 & 62207747 & $\mathrm{C}$ & A & Non-SNV & p.T669N & $\mathrm{N}$ & $\mathrm{U}$ \\
\hline HLF & CGC & 17 & 53345172 & $\mathrm{~T}$ & $\mathrm{C}$ & Non-SNV & p.V59A & $\mathrm{N}$ & $\mathrm{D}$ \\
\hline KMT2D & CGC & 12 & 49446404 & $\mathrm{C}$ & $\mathrm{T}$ & Non-SNV & p.V401M & $\mathrm{N}$ & $\mathrm{P}$ \\
\hline MLLT3 & CGC & 9 & 20414344 & CTG & - & Non-FS DEL & p.163_164del & $\mathrm{Y}$ & $\mathrm{U}$ \\
\hline MN1 & CGC & 22 & 28194933 & - & $\begin{array}{l}\text { TGCTGC } \\
\text { TGCTGC }\end{array}$ & $\begin{array}{l}\text { Non-FS } \\
\text { INS }\end{array}$ & $\begin{array}{l}\text { p.Q533delins } \\
\text { QQQQQ }\end{array}$ & $\mathrm{N}$ & $\mathrm{U}$ \\
\hline NKX2-1 & CGC & 14 & 36986635 & $\mathrm{C}$ & $\mathrm{T}$ & Non-SNV & p.G322S & $\mathrm{N}$ & $\mathrm{D}$ \\
\hline NRG1 & CGC & 8 & 32505286 & $\mathrm{C}$ & $\mathrm{G}$ & Non-SNV & p.S17C & $\mathrm{N}$ & $\mathrm{D}$ \\
\hline PRDM2 & CGC & 1 & 14107360 & $\mathrm{~T}$ & $\mathrm{~A}$ & Non-SNV & p.S823T & $\mathrm{Y}$ & $\mathrm{D}$ \\
\hline PTPRC & CGC & 1 & 198675962 & $\mathrm{~A}$ & $\mathrm{G}$ & Non-SNV & p.N101S & $\mathrm{N}$ & $\mathrm{U}$ \\
\hline RAD51D & CPG & 17 & 33428245 & G & A & Non-SNV & p.A181V & $\mathrm{N}$ & $\mathrm{P}$ \\
\hline RNF213 & CGC & 17 & 78346531 & $\mathrm{C}$ & $\mathrm{A}$ & Non-SNV & p.P4250T & $\mathrm{N}$ & $\mathrm{U}$ \\
\hline ROS1 & CGC & 6 & 117662652 & $\mathrm{C}$ & $\mathrm{G}$ & Non-SNV & p.E1605Q & $\mathrm{N}$ & $\mathrm{P}$ \\
\hline WNK2 & CGC & 9 & 96015284 & $\mathrm{~T}$ & $\mathrm{C}$ & Non-SNV & p.C652R & $\mathrm{N}$ & $\mathrm{D}$ \\
\hline
\end{tabular}

Non-SNV, non-synonymous single nucleotide variation; non-FS DEL, non-frameshift deletion; non-FS INS, non-frameshift insertion; CGC, Cancer Gene Census; CPG, Cancer Predisposition Genes; P, possibly damaging; D, deleterious; U, uncertain significance; Y, yes; N, no; AA, amino acid.

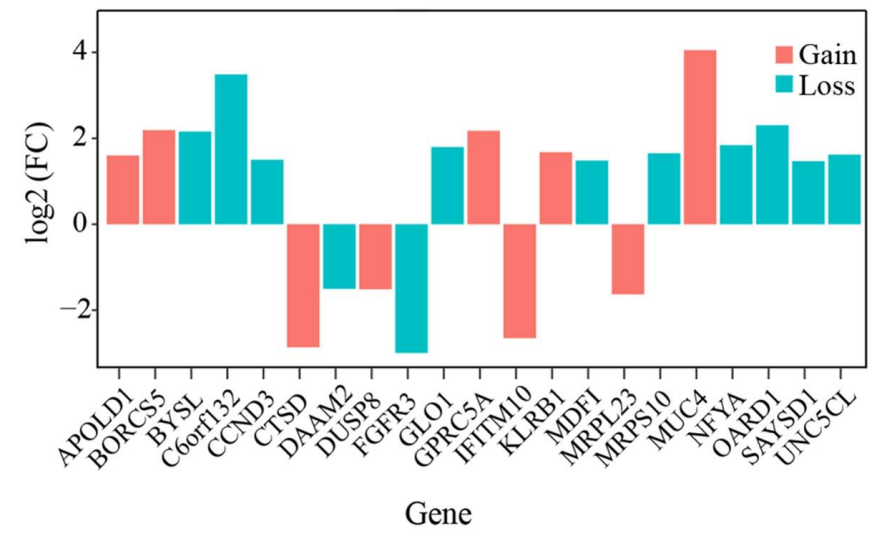

Figure 1. Differentially expressed genes with structural alternations. Structural variation and copy number alternation analysis identified 21 genes, which were differentially expressed. The angular axis shows $\log _{2}$ fold change between tumor and adjacent normal tissues.

with $\mathrm{P} \leq 0.001$ and absolute $\log _{2}$ fold change value $\geq 1$ were considered as significantly differentially expressed genes. In total, 766 down- and 765 upregulated genes were obtained (Fig. S1B). The RNA-Seq result was subsequently matched to the whole genome sequencing data for further analysis.

Germline mutation analysis. Germline mutation analysis revealed a total of 4,228,339 single nucleotide polymorphisms (SNPs) and Indels with $95.03 \%$ of the sites previously reported in the dbSNP database. The transition to transversion ratio was 2.05 , indicating that the germline analysis had high confidence, as the empirical value for whole genome analysis

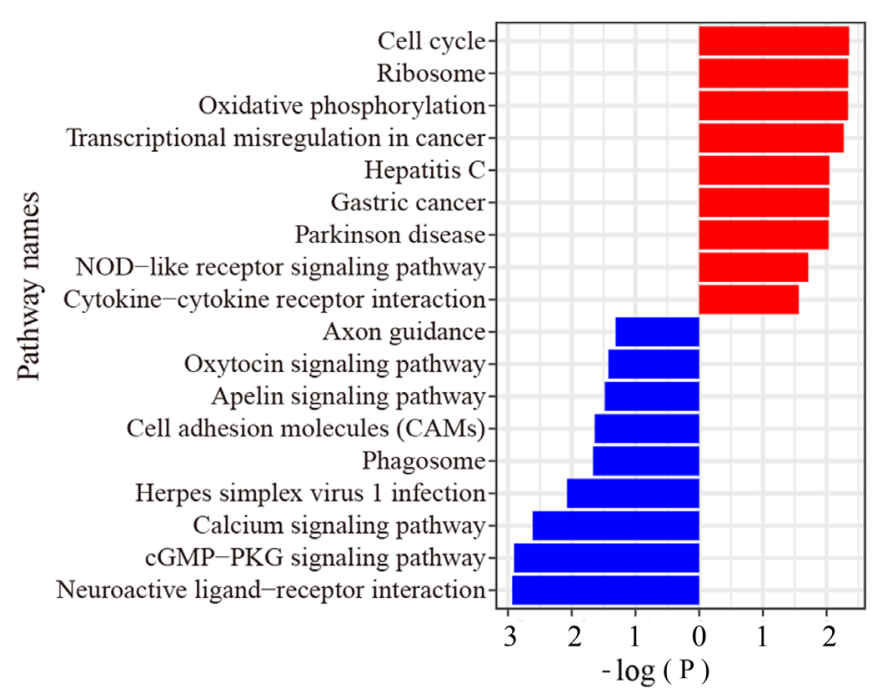

Figure 2. Gene Set Enrichment Analysis revealed dysregulated pathways in RNA-seq. Upregulated genes are indicated by blue and the downregulated by red.

is $\sim 2.1$ (31). Among the germline variants, $21,405 \mathrm{SNPs} /$ Indels were located in the exonic regions, of which 10,136 were predicted to alter the protein, including 9,504 non-synonymous single nucleotide variations (SNVs), 112 frameshift deletions, 90 frameshift insertions, 173 non-frameshift deletions, 169 non-frameshift insertions, 78 stop-gain, and 10 stop-loss. More than $1 \%$ minor allele frequency variants from publicly available databases were removed, as those sites are common in the population. Of the candidate genes from the CGC and CPG databases, 13 SNPs and two Indels were identified 

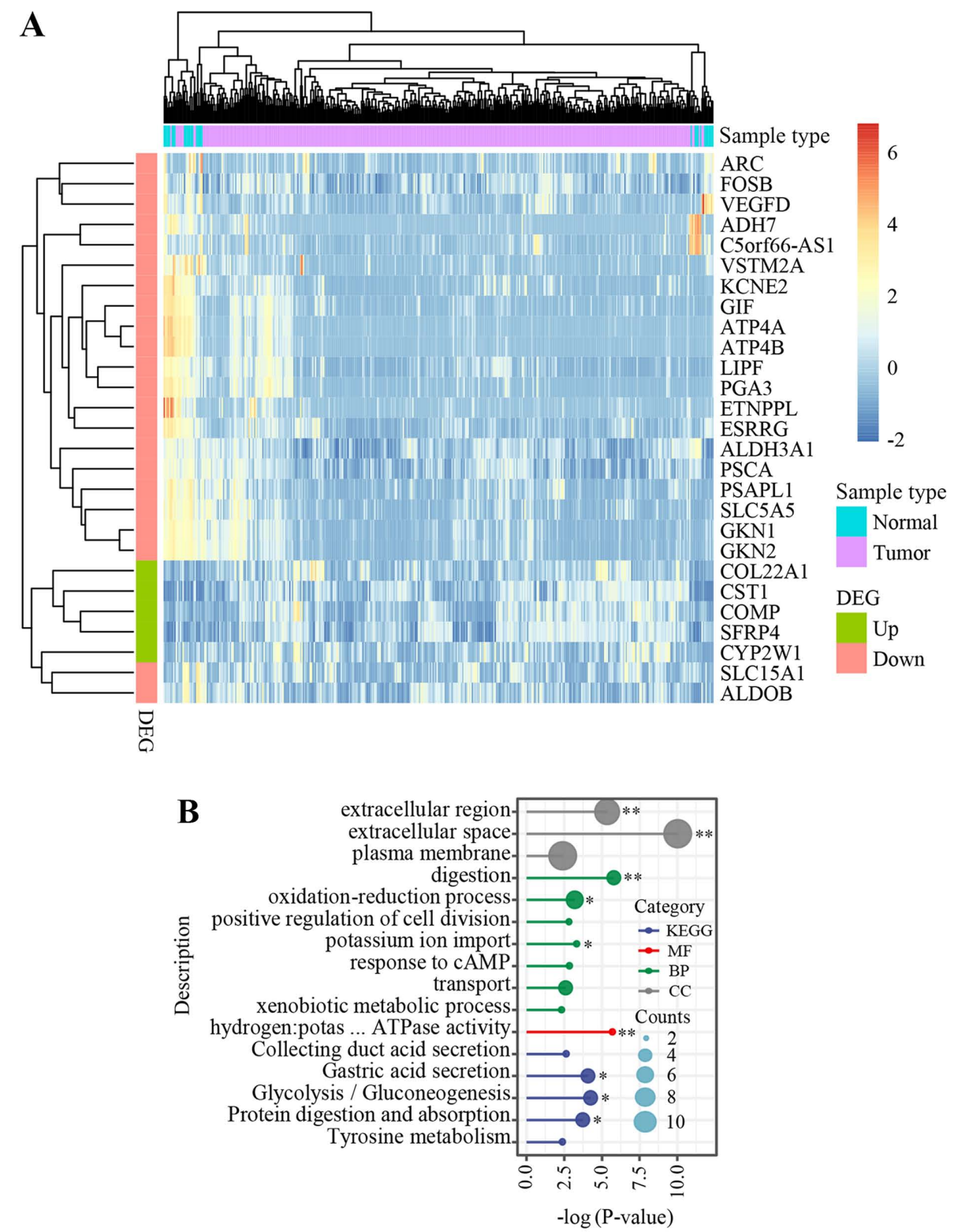

Figure 3. Genomic features in the young patient in the present study compared with that in patients from TCGA STAD dataset. (A) Heatmap of the 27 genes, which were dysregulated in both TCGA STAD and in the present study. Counts per million (CPM) indicates the expression level of each gene and log $(\mathrm{CPM}+1)$, which ranged from -2 (blue) to 6 (red), was used for sample clustering and visualization. (B). Gene Ontology enrichment analysis and KEGG pathway analysis identified several significant pathways. ${ }^{*} \mathrm{P}<0.05$ and ${ }^{* *} \mathrm{P}<0.01$. DEG, differentially expressed gene; KEGG, Kyoto Encyclopedia of Genes and Genomes; MF, molecular functions; CC, cellular components; BP, biological processes; TCGA, The Cancer Genome Atlas; STAD, stomach adenocarcinoma; hydrogen:potas ... ATPase activity, hydrogen:potassium-exchanging ATPase activity.

in 15 genes (Table I). A deleterious score evaluation of the 15 variants revealed that mutations in $D O C K 8$ (p.D63N), $H L F$ (p.V59A), NKX2-1 (p.G322S), NRG1 (p.S17C), PRDM2 (p.S823T), WNK2 (p.C652R) were deleterious, while mutations in KMT2D (p.V401M), RAD51D (p.A181V) and ROSI (p.E1605Q) were possibly damaging.

Somatic mutation analysis. A total of 3 softwares from the Broad Institute were used to identify the somatic variants and 114 variants in the exonic regions were found. This included 45 non-synonymous SNVs, 6 frameshift deletions, 11 frameshift insertions, 17 non-frameshift deletions, 3 non-frameshift insertions, 2 stop-gain and 30 synonymous SNVs. Similar to the germline mutation analysis, common variants were filtered out with publicly available datasets to select the most infor- mative somatic variants. In total, 29 variants were obtained, including 13 mutations which were reported in the Catalogue of Somatic Mutations in Cancer database (Table SI). Of these, 3 protein-truncating variants (BPNT1, p.S144I; FRG1, p.N153D; and TAS2R31, p.L59F) were predicted to be deleterious and one variant (KRTAP5-3, p.C185S) was predicted to be possibly damaging. However, for transcriptional regulation, 3 genes (MUC2, MUC4 and SLC8A2) were found to be differentially expressed.

Large-scale variation analysis. To identify large-scale variations in the patient, the copy number alterations and complex structural events in the tumor sample were investigated. The structural variant discovery tool, DELLY, reported 701 structural events; however, only in-frame events or fusions 
over $50 \mathrm{~kb}$ between two genes were retained. Ultimately, 20 significant structural variations were reported (Table SII). Another sensitive tool, FACETS, detected 58 large segments, of which 62 were deletions and 112 were duplications found in the coding regions (Table SIII). Combining the results from the two tools, nine genes (CDKN1B, FBXW7, MUC4, CCND3, ETV6, FGFR2, FGFR3, TFEB and KMT2C) were reported in either the CGC or CPG databases. CDKNIB, FBXW7 and $M U C 4$ showed duplications, and there were deletions in CCND3, ETV6, FGFR2, FGFR3 and TFEB. Although KMT2C rearranged with $B A G E 2$, the latter was not reported in the 2 databases. The mRNA expression levels of the genes with structural variations was also investigated and 21 were found to be differentially expressed in the tumor sample (Fig. 1).

Integrated pathway analysis results. RNA-Seq and mutation data were analyzed to characterize the genomic alterations in known signaling pathways. The GO enrichment for somatic mutated genes revealed terms, which included 3 mucin family genes, $M U C 2$, , MUC4 and MUC6, and were enriched in 'maintenance of gastrointestinal epithelium', 'O-glycan processing', and digestive system process, while KRTAP5-3, TCHH, RPTN, $P O U 3 F 1$ were enriched in epidermal cell differentiation pathway (Table SIV). The KEGG enrichment analysis revealed SLC8A2 and SLC25A5 were enriched in calcium signaling and cGMP-PKG signaling pathways (Table SIV). From the RNA-Seq data, GSEA showed that the upregulated genes were enriched in 'cGMP-PKG signaling', 'calcium signaling', and 'oxytocin signaling' pathways, while the downregulated genes were enriched in 'cell cycle' pathway, 'oxidative phosphorylation' pathway, 'transcriptional mis-regulation in cancer' pathway and 'GC pathway' (Fig. 2).

Comparison with TCGA STAD GC features. To ensure the present study was representative and valuable, the genomic features of the patient was compared with data from TCGA STAD dataset, which was downloaded from TCGAbiolinks, and 329 (136 up- and 193 downregulated genes) differentially expressed genes were identified, which were also found in the RNA-Seq analysis. A total of three tools were used to identify the most significantly differentially expressed genes, and 136 up- and 193 downregulated genes were identified (Fig. S2A), and were subsequently investigated in the tumor tissue sample obtained from the patient with GC and compared with that in the adjacent normal tissue. In total, 27 genes were identified, including five that were up- and 22 that were downregulated (Fig. 3A). GO and KEGG pathway enrichment analysis revealed one (GO:MF) and three (KEGG) significant terms, respectively, including digestion and gastric acid secretion (Fig. 3B).

To determine the associated functional genomic alterations of these 27 genes, the previous studies that reported them were analyzed. In the present study, there were no somatic mutations in the 27 genes, however, 12 of the genes, including gastrokine 2 (GKN2), prosaposin-like 1 (PSAPL1), ethanolamine-phosphate phospho-lyase (ETNPPL), cytochrome $\mathrm{P} 450$ family 2 subfamily $\mathrm{W}$ member 1 ( $C Y P 2 W 1), S F R P 4$, collagen type XXII $\alpha 1$ chain, prostate stem cell antigen, lipase $\mathrm{F}$, gastric type, solute carrier family 15 member 1 (SLC15Al), ATPase $\mathrm{H}+/ \mathrm{K}+$ transporting subunit $\beta$ (ATP4B), aldehyde dehydrogenase 3 family member A1 (ALDH3Al) and cystatin SN (CST1) were mutated in germline. Notably, two (SFRP4 and $S L C 15 A 1)$ of the 12 germline mutated genes have been reported in previous studies. Germline mutations in SFRP4 (P320T and R340K) were reported to be strongly associated with human cancer types (45) while mutations in SLC15A1 were linked with bowel diseases (46).

The KM plotter database was subsequently used with the log-rank test to investigate the association between the expression level of the 27 genes and clinical outcomes (both disease-free and overall survival times). In the database, patients with GC are divided into high and low expression groups, with each percentile of mRNA expression between the lower $(25 \%)$ and upper quartiles $(75 \%)$ used as a cut-off point. A total of 10 genes $(A L D H 3 A 1, E T N P P L$, activity regulated cytoskeleton associated protein $(A R C), A T P 4 B$, CST1, CYP2W1, GKN2, PSAPL1, SFRP4 and SLC15A1) with differential expression were significantly associated $(\mathrm{P}<0.05)$ with survival times. In all the 10 genes, those with high expression (red curve) were correlated with a poorer survival while genes with low expression (black curve) correlated with higher survival in patients with GC (Fig. S2B).

\section{Discussion}

In an attempt to improve the understanding of the pathobiology of GC in young patients and provide insights for personalized treatment strategies, the genome of a 26-year old patient with GC was performed using both whole genome and whole transcriptome sequencing. Germline and somatic mutations analyses of the whole genome sequencing data revealed 15 cancer related genes with germline mutations, 27 genes with 29 informative somatic mutations and nine genes with structural events that could underlie the progression of GC in the patient. Of the 27 genes containing somatic mutations, 26 have not been previously reported as driver genes in TCGA (42). Only MUC6, a immunohistochemical marker used to support the diagnosis of gastric-type EA (47), has been reported as a driver gene. In the mutation significance analysis, a set of scoring system was used to predict deleterious amino acid substitutions. This analysis revealed nine germline variants and four somatic mutations with potentially pathogenic sites. Compared with somatic mutations, more deleterious germline sites were detected and genes containing these sites were all found to be cancer-associated, suggesting that inheritance disorders might be a key risk factor for the development of GC in the patient.

To improve the understanding of the inter-relationships between genomic alterations and the transcriptome, the mutated genes at the transcriptional level were also investigated. It was found that genes with somatic mutations, such as MUC2, MUC4, $S L C 8 A 2$, and with somatic structural mutations, such as $C C N D 3$, FGFR2 and FGFR3, were differentially expressed, suggesting that they could be promising precision therapy targets.

Mucin genes are known to maintain the gastrointestinal epithelium, and persistent mucosal inflammation increase the risk of developing GC (48). In addition, the fibroblast growth factor receptor (FGFR) pathway plays a key role in GC pathogenesis, and detection of FGFR2 copy number in the plasma circulating tumor DNA are potential predictive biomarkers to FGFR inhibition (49). 
With respect to heterogeneity of GC, it was hypothesized that mutations in different genes that are involved in the same pathway could result in similar clinical phenotypes. Therefore, different pathways were investigated that could be dysregulated in the patient with GC. The calcium signaling pathway, cGMP-PKG signaling pathway and transcriptional mis-regulation were enriched at both the genomic and transcriptomic levels, suggesting that these pathways might play crucial roles in young patients with GC. Notably, these pathways were not found from the analysis of TCGA STAD dataset (42), suggesting that the pathogenesis of GC in young individuals, particularly in the patient in the present study may be different. Nevertheless, the genomic features of the young patient with GC was compared with TCGA STAD data and 27 differential expression genes were identified, particularly germline mutations in SFRP4 (P320T and R340K), which are strongly associated with human cancers (45). SFRP4 is a member of the SFRP family (50). SFRP4s contain a cysteine-rich domain homologous to the putative Wnt-binding site of Frizzled proteins (50) and act as soluble modulators of Wnt signaling, which is a well-known pathway that is involved in tumorigenesis of GC (51). This indicates that such germline mutations could be informative in designing patient specific therapies. Several germline mutations were found in the 27 genes; however, no somatic alterations or structural events were identified, suggesting that genetic factors may be important in the development of early GC.

Notably, the youngest patient in TCGA STAD dataset was 30-years-old and $>99 \%(439 / 443)$ of the patients were $>40$ years old, thus, TCGA STAD data could have originated from elderly patients with GC. This supports the requirement to investigate the genome in younger patients with GC to identify possible therapeutic targets. The patient in the present study was diagnosed at age 26 , which was much younger compared with patients in TCGA STAD dataset. TCGA-STAD RNA-seq data was reanalyzed and 331 dysregulated genes were found (138 were up- and 193 were downregulated), however, only 27 of these were found in the patient in the present study, suggesting that there was a considerable difference between GC in young and elderly patients. There may be indications that the pathogenesis of the patient was substantially different from typical patients with GC, as cancer-related pathways, which were significantly enriched at both genomic and transcriptomic levels in the patient were also not found in TCGA STAD dataset.

In conclusion, genes with germline (SFRP4), and somatic mutations (MUC2, MUC4, SLC8A2), and those with structural variations (CCND3, FGFR2 and FGFR3), which were differentially expressed in the patient could be promising precision therapy targets.

\section{Acknowledgements}

Not applicable.

\section{Funding}

This study was supported in part by the Hefei National Laboratory for Physical Sciences at Microscale (grant no. HFNLM20180012).

\section{Availability of data and materials}

We have deposited the data to National Genomics Data Center with the project number PRJCA002949. The datasets are not publicly accessible due to local data protection laws. However, access to the data can be granted upon a reasonable request to the corresponding author.

\section{Authors' contributions}

QW and KH conceived the study and designed the experiments. LL and ZH provided the tissue samples and supplied the clinical and pathological information of the patient. QR, OEA and SW performed sample preparation, DNA isolation for sequencing and library construction. KH and WY performed the bioinformatics analysis. OEA, WY, KH and QW drafted and revised the manuscript and supplementary information. All authors approved the final version of the manuscript.

\section{Ethics approval and consent to participate}

This study was approved by the Ethics Committee of the First Affiliated Hospital of Anhui Medical University. The patient provided written informed consent for the use of the resected tissue, and the sample was obtained under Institutional Review Board approval.

\section{Patient consent for publication}

Not applicable.

\section{Competing interests}

The authors declare that they have no competing interests.

\section{References}

1. De Vita F, Di Martino N, Fabozzi A, Laterza MM, Ventriglia J, Savastano B, Petrillo A, Gambardella V, Sforza V, Marano L, et al: Clinical management of advanced gastric cancer: The role of new molecular drugs. World J Gastroentero 20: 14537-14558, 2014.

2. Mori M, Sugimachi K, Ohiwa T, Okamura T, Tamura S and Inokuchi K: Early gastric carcinoma in Japanese patients under 30 years of age. Br J Surg 72: 289-291, 1985.

3. Lim S, Lee HS, Kim HS, Kim YI and Kim WH: Alteration of E-cadherin-mediated adhesion protein is common, but microsatellite instability is uncommon in young age gastric cancers. Histopathology 42: 128-136, 2003.

4. Smith BR and Stabile BE: Extreme aggressiveness and lethality of gastric adenocarcinoma in the very young. Arch Surg 144: 506-510, 2009.

5. Quijano Orvananos F, Moreno Paquentin E, Alvarez JJ, Martinez Munive A and Butron Perez L: Gastric carcinoma in patients under 35 years. Rev Gastroenterol Mex 64: 75-77, 1999 (In Spanish).

6. Theuer CP, Kurosaki T, Taylor TH and Anton-Culver H: Unique features of gastric carcinoma in the young: A population-based analysis. Cancer 83: 25-33, 1998.

7. Wierinckx A, Roche M, Raverot G, Legras-Lachuer C, Croze S, Nazaret N, Rey C, Auger C, Jouanneau E, Chanson P, et al: Integrated genomic profiling identifies loss of chromosome 11p impacting transcriptomic activity in aggressive pituitary PRL Tumors. Brain Pathol 21: 533-543, 2011

8. Cancer Genome Atlas Research Network; Bass AJ, Thorsson V, Shmulevich I, Reynolds SM, Miller M, Bernard B, Hinoue T, Laird PW, Curtis C, et al: Comprehensive molecular characterization of gastric adenocarcinoma. Nature 513: 202-209, 2014.

9. Chmielecki J and Meyerson M: DNA sequencing of cancer: What Have We Learned? Annu Rev Med 65: 63-79, 2014. 
10. Garraway LA and Lander ES: Lessons from the Cancer Genome. Cell 153: 17-37, 2013.

11. Vogelstein B,Papadopoulos N, Velculescu VE,Zhou SB, Diaz LA and Kinzler KW: Cancer genome landscapes. Science 339: 1546-1558, 2013.

12. Garraway LA: Genomics-driven oncology: Framework for an emerging paradigm. J Clin Oncol 31: 1806-1814, 2013.

13. Chen R: Abstract 4495: Development and clinical application of an integrative genomic approach to personalized cancer therapy. Cancer Res: 76, 2016 doi: 10.1158/1538-7445.AM2016-4495.

14. Edge SB and Compton CC: The American Joint Committee on Cancer: The 7th edition of the AJCC cancer staging manual and the future of TNM. Ann Surg Oncol 17: 1471-1474, 2010.

15. Krueger F: Trim Galore: A wrapper tool around Cutadapt and FastQC to consistently apply quality and adapter trimming to FastQ files, with some extra functionality for MspI-digested RRBS-type (Reduced Representation Bisufite-Seq) libraries, 2012

16. Dobin A, Davis CA, Schlesinger F, Drenkow J, Zaleski C, Jha S, Batut P, Chaisson M and Gingeras TR: STAR: Ultrafast universal RNA-seq aligner. Bioinformatics 29: 15-21, 2013.

17. Anders S, Pyl PT and Huber W: HTSeq-a Python framework to work with high-throughput sequencing data. Bioinformatics 31 : $166-169,2015$

18. Robinson MD, McCarthy DJ and Smyth GK: edgeR: A Bioconductor package for differential expression analysis of digital gene expression data. Bioinformatics 26: 139-140, 2010.

19. Li H: Aligning sequence reads, clone sequences and assembly contigs with BWA-MEM. (Submitted on 16 Mar 2013 (v1), last revised 26 May, 2013 (this version, v2)).

20. DePristo MA, Banks E, Poplin R, Garimella KV, Maguire JR, Hartl C, Philippakis AA, del Angel G, Rivas MA, et al: A framework for variation discovery and genotyping using next-generation DNA sequencing data. Nat Genet 43: 491-498, 2011.

21. Poplin R, Ruano-Rubio V, DePristo MA, Fennell TJ, Carneiro MO, Van der Auwera GA, Kling DE, Gauthier LD Levy-Moonshine A, Roazen D, et al: Scaling accurate genetic variant discovery to tens of thousands of samples. BioRxiv: July 24, 2017 doi: https://doi.org/10.1101/201178.

22. Cibulskis K, Lawrence MS, Carter SL, Sivachenko A, Jaffe D, Sougnez C, Gabriel S, Meyerson M, Lander ES and Getz G: Sensitive detection of somatic point mutations in impure and heterogeneous cancer samples. Nat Biotechnol 31: 213-219, 2013.

23. Colaprico A, Silva TC, Olsen C, Garofano L, Cava C, Garolini D, Sabedot TS, Malta TM, Pagnotta SM, Castiglioni I, et al: TCGAbiolinks: An R/Bioconductor package for integrative analysis of TCGA data. Nucleic Acids Res 44: e71, 2016.

24. 1000 Genomes Project Consortium; Auton A, Brooks LD Durbin RM, Garrison EP, Kang HM, Korbel JO, Marchini JL, McCarthy S, McVean GA and Abecasis GR: A global reference for human genetic variation. Nature 526: 68-74, 2015.

25. Karczewski KJ, Weisburd B, Thomas B, Solomonson M Ruderfer DM, Kavanagh D, Hamamsy T, Lek M, Samocha KE, Cummings BB, et al: The ExAC browser: Displaying reference data information from over 60000 exomes. Nucleic Acids Res 45: D840-D845, 2016.

26. Karczewski KJ and Karczewski LF: The genome aggregation database (gnomAD), 2017.

27. Liu X, Wu C, Li C and Boerwinkle E: dbNSFP v3.0: A One-stop database of functional predictions and annotations for human nonsynonymous and splice-site SNVs. Hum Mutat 37: 235-241, 2016.

28. Sherry ST, Ward MH, Kholodov M, Baker J, Phan L, Smigielski EM and Sirotkin K: dbSNP: The NCBI database of genetic variation. Nucleic Acids Res 29: 308-311, 2001.

29. Tate JG, Bamford S, Jubb HC, Sondka Z, Beare DM, Bindal N, Boutselakis H, Cole CG, Creatore C, Dawson E, et al: COSMIC: The catalogue of somatic mutations in cancer. Nucleic Acids Res 47: D941-D947, 2019.

30. Kimura M: A simple method for estimating evolutionary rates of base substitutions through comparative studies of nucleotide-sequences. J Mol Evol 16: 111-120, 1980.

31. Mwenifumbo JC and Marra MA: Cancer genome-sequencing study design. Nat Rev Genet 14: 321-332, 2013.
32. Shen R and Seshan VE: FACETS: Allele-specific copy number and clonal heterogeneity analysis tool for high-throughput DNA sequencing. Nucleic Acids Res 44: e131, 2016.

33. Team RC: R: A language and environment for statistical computing, 2013.

34. Rausch T, Zichner T, Schlattl A, Stutz AM, Benes V and Korbel JO DELLY: Structural variant discovery by integrated paired-end and split-read analysis. Bioinformatics 28: i333-i339, 2012.

35. Dong C, Wei P, Jian X, Gibbs R, Boerwinkle E, Wang K and Liu X: Comparison and integration of deleteriousness prediction methods for nonsynonymous SNVs in whole exome sequencing studies. Hum Mol Genet 24: 2125-2137, 2015.

36. Futreal PA, Coin L, Marshall M, Down T, Hubbard T, Wooster R, Rahman N and Stratton MR: A census of human cancer genes. Nat Rev Cancer 4: 177-183, 2004.

37. Rahman N: Realizing the promise of cancer predisposition genes. Nature 505: 302-308, 2014.

38. Yu GC, Wang LG, Han YY and He QY: clusterProfiler: An R package for comparing biological themes among gene clusters. OMICS 16: 284-287, 2012.

39. Subramanian A, Tamayo P, Mootha VK, Mukherjee S, Ebert BL Gillette MA, Paulovich A, Pomeroy SL, Golub TR, Lander ES and Mesirov JP: Gene set enrichment analysis: A knowledge-based approach for interpreting genome-wide expression profiles. Proc Natl Acad Sci USA 102: 15545-15550, 2005.

40. Ritchie ME, Phipson B, Wu D, Hu Y, Law CW, Shi W and Smyth GK: limma powers differential expression analyses for RNA-sequencing and microarray studies. Nucleic Acids Res 43: e47, 2015 .

41. Love MI, Huber W and Anders S: Moderated estimation of fold change and dispersion for RNA-seq data with DESeq2. Genome Biol 15: 550, 2014.

42. Bailey MH, Tokheim C, Porta-Pardo E, Sengupta S, Bertrand D, Weerasinghe A, Colaprico A, Wendl MC, Kim J, Reardon B, et al: Comprehensive characterization of cancer driver genes and mutations. Cell 174: 1034-1035, 2018.

43. Szász AM, Lánczky A, Nagy Á, Förster S, Hark K, Green JE, Boussioutas A, Busuttil R, Szabó A and Győrffy B: Cross-validation of survival associated biomarkers in gastric cancer using transcriptomic data of 1,065 patients. Oncotarget 7: 49322-49333, 2016.

44. Taiaroa G, Rawlinson D, Featherstone L, et al: Direct RNA sequencing and early evolution of SARS-CoV-2. bioRxiv, 2020 (Epub ahead of print)

45. He ML, Chen Y, Chen Q, He Y, Zhao J, Wang J, Yang H and Kung HF: Multiple gene dysfunctions lead to high cancer-susceptibility: Evidences from a whole-exome sequencing study. Am J Cancer Res 1: 562-573, 2011.

46. Zucchelli M, Torkvist L, Bresso F, Halfvarson J, Hellquist A, Anedda F, Assadi G, Lindgren GB, Svanfeldt M, Janson M, et al: PepT1 oligopeptide transporter (SLC15A1) gene polymorphism in inflammatory bowel disease. Inflamm Bowel Dis 15: $1562-1569,2009$.

47. Hodgson A,Parra-HerranCand Mirkovic J:Immunohistochemical expression of HIK1083 and MUC6 in endometrial carcinomas. Histopathology 75: 552-558, 2019.

48. Li M, Huang L, Qiu H, Fu Q, Li W, Yu Q, Sun L, Zhang L, Hu G, $\mathrm{Hu} \mathrm{J}$ and Yuan X: Helicobacter pylori infection synergizes with three inflammation-related genetic variants in the GWASs to increase risk of gastric cancer in a Chinese population. PLoS One 8: e74976, 2013

49. Hierro C, Alsina M, Sanchez M, Serra V, Rodon J and Tabernero J: Targeting the fibroblast growth factor receptor 2 in gastric cancer: Promise or pitfall? Ann Oncol 28: 1207-1216, 2017.

50. Cruciat CM and Niehrs C: Secreted and transmembrane wnt inhibitors and activators. Cold Spring Harb Perspect Biol 5: a015081, 2013.

51. Herr P, Hausmann G and Basler K: WNT secretion and signalling in human disease. Trends Mol Med 18: 483-493, 2012.

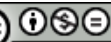

This work is licensed under a Creative Commons Attribution-NonCommercial-NoDerivatives 4.0 International (CC BY-NC-ND 4.0) License. 